\title{
Peptide-coated gold nanoparticles for modulation of angiogenesis in vivo
}

\author{
This article was published in the following Dove Press journal: \\ International Journal of Nanomedicine \\ 7 June 2016 \\ Number of times this article has been viewed
}

\author{
Catarina Roma-Rodrigues \\ Amelie Heuer-Jungemann² \\ Alexandra R Fernandes' \\ Antonios G Kanaras ${ }^{2}$ \\ Pedro V Baptista' \\ 'UCIBIO, Departamento de Ciências \\ da Vida, Faculdade de Ciências e \\ Tecnologia, Universidade NOVA \\ de Lisboa, Caparica, Portugal; \\ ${ }^{2}$ Institute for Life Sciences, Physics \\ and Astronomy, Faculty of Physical \\ Sciences and Engineering, University \\ of Southampton, Southampton, UK
}

Correspondence: Pedro V Baptista UCIBIO, Departamento de Ciências da Vida, Faculdade de Ciências e Tecnologia, Universidade NOVA de Lisboa, Campus de Caparica, 2829-5 I6, Caparica, Portugal Tel +35 I $2 / 2948530$

Fax +35I 212948530

Email pmvb@fct.unl.pt

\begin{abstract}
In this work, peptides designed to selectively interact with cellular receptors involved in the regulation of angiogenesis were anchored to oligo-ethylene glycol-capped gold nanoparticles (AuNPs) and used to evaluate the modulation of vascular development using an ex ovo chick chorioallantoic membrane assay. These nanoparticles alter the balance between naturally secreted pro- and antiangiogenic factors, under various biological conditions, without causing toxicity. Exposure of chorioallantoic membranes to AuNP-peptide activators of angiogenesis accelerated the formation of new arterioles when compared to scrambled peptidecoated nanoparticles. On the other hand, antiangiogenic AuNP-peptide conjugates were able to selectively inhibit angiogenesis in vivo. We demonstrated that AuNP vectorization is crucial for enhancing the effect of active peptides. Our data showed for the first time the effective control of activation or inhibition of blood vessel formation in chick embryo via AuNP-based formulations suitable for the selective modulation of angiogenesis, which is of paramount importance in applications where promotion of vascular growth is desirable (eg, wound healing) or ought to be contravened, as in cancer development.
\end{abstract}

Keywords: angiogenesis activators, antiangiogenic, CAM assay, gold nanoparticles, peptidecoated gold nanoparticles, vascular development

\section{Introduction}

Angiogenesis, or the formation of new capillary blood vessels, occurs mainly during mammalian development and reproduction but has also been associated with several pathological conditions, including cancer. Neoangiogenesis establishes one of the first steps of tumor progression; it is the process by which the nutrients and gases needed for sustained and aggressive growth of tumor cells, invasion, and metastasis are exchanged through blood vessels. Thus, tumor angiogenesis constitutes an important point of control of cancer progression. ${ }^{1}$ Various antiangiogenic drugs designed to prevent endothelial cell proliferation have been reported. ${ }^{1}$ Despite a few successful results, downfalls of these drugs often include the need for introduction of large doses of drugs leading to undesired toxic side effects. An effective method of dose reduction is the conjugation of drugs to nanoparticles (NPs).

Gold nanoparticles (AuNPs) are the proposed ideal theranostic vehicles for cancer. The tumor environment presents an enhanced permeability and retention effect, which results from the angiogenic nature of the tumor tissue and its ability to sequester chemotherapeutic agents from healthy tissues. ${ }^{2,3}$ Ideal drug delivery nanosystems may exploit this enhanced permeability and retention effect, accumulate in the tumor tissue, and release the desired cargo where needed at a high concentration, thus reducing the undesirable deleterious effects to healthy tissue., 


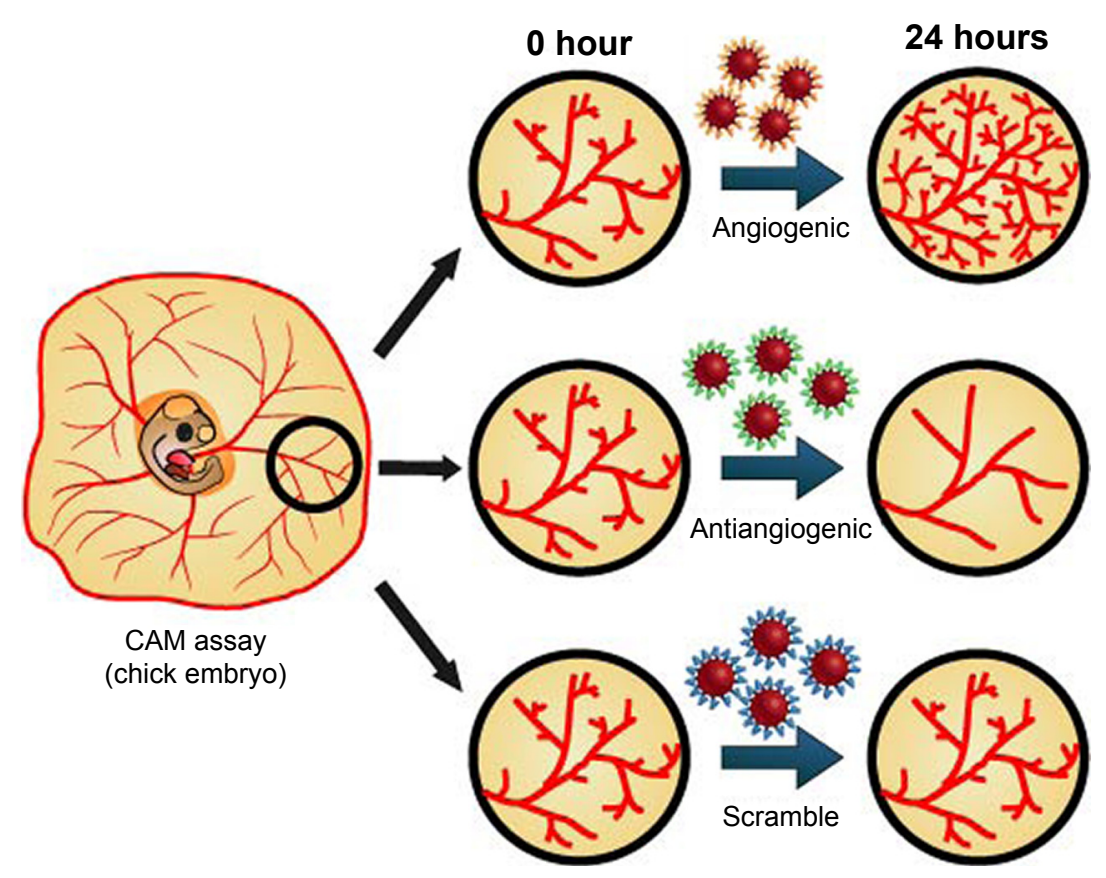

Figure I Peptide-coated AuNPs for in vivo targeting of angiogenesis.

Notes: CAM assay was performed with AuNPs functionalized with three different peptides: proangiogenic; antiangiogenic, and a scrambled peptide as control. Abbreviations: AuNPs, gold nanoparticles; CAM, chorioallantoic membrane.

Additional targeting of malignant cells may be provided by small molecules, such as cell-penetrating peptides, that are able to cross cellular membranes, improving intracellular delivery of NPs. ${ }^{6-8}$

The activation or inhibition of in vitro angiogenesis using functional peptide-coated AuNPs has been previously demonstrated. ${ }^{9}, 10$ The peptides, anchored to oligo-ethylene glycol (OEG)-capped gold nanospheres, were designed to selectively interact with cellular receptors involved in the regulation of angiogenesis: P1 (KPQPRPLS) binds to the vascular endothelial growth factor receptor-1 and promotes signal cascade activation of angiogenic genes; P2 (KPRQPSLP) does not interact with any receptor and is taken up by the cells (scrambled), and P3 (KATWLPPR) binds to neuropilin-1 receptor and promotes receptor internalization. ${ }^{9,10}$ These NPs alter the balance between naturally secreted pro- and antiangiogenic factors under various biological conditions without causing toxicity. ${ }^{9}$

The use of models, which could mimic a biological system as closely as possible, is crucial. The chorioallantoic membrane (CAM) assay is one of the most used in vivo model systems for angiogenesis-related studies. The CAM is a highly vascularized tissue of the avian embryo and serves as an excellent in vivo model for screening potential novel therapeutics ${ }^{11}$ and studying the effects of various drugs/ molecules on neoangiogenesis. ${ }^{12}$ Ex ovo strategies enhance the accessibility of the CAM and chick embryo, facilitating experimental manipulation of the embryo ${ }^{13}$ and enabling the in vivo documentation of angiogenesis-related studies.

Here, we use the same three types of peptide-coated NPs mentioned previously to evaluate the effect in in vivo angiogenesis, and demonstrate for the first time, the effective control of activation or inhibition of blood vessel formation in chick embryos (Figure 1).

\section{Materials and methods}

\section{Materials}

All reagents were of analytical grade and purchased from SigmaAldrich (St Louis, MO, USA). Milli-Q water (EMD Millipore, Billerica, MA, USA) was used in all experiments. Fertilized eggs were acquired from Avibom, Lisboa, Portugal. Peptides (lyophilized precipitated) were obtained from Peptide Synthetic, Funtley, UK and used without further purification.

\section{Synthesis of AuNPs}

Gold nanospheres ( $13 \pm 2 \mathrm{~nm}$ ) were synthesized according to procedures mentioned in the literature. ${ }^{14}$ Briefly, an aqueous solution of sodium tetrachloroaurate $(100 \mathrm{~mL}, 1 \mathrm{mM})$ was brought to the boil under stirring. Once boiling, a hot aqueous solution of trisodium citrate $(5 \mathrm{~mL}, 2 \% \mathrm{w} / \mathrm{v})$ was added to the gold solution. A color change from yellow to colorless to deep red indicated the formation of NPs. The solution 
was then stirred under boiling for an additional 15 minutes and subsequently allowed to cool to room temperature under stirring. Prepared AuNPs were filtered $(0.2 \mu \mathrm{m}$ syringe filter $)$ and subsequently stored at $4{ }^{\circ} \mathrm{C}$.

\section{Surface capping of AuNPs with OEG}

AuNPs were then functionalized with OEG, monocarboxy (1-mercaptoundec-11-yl) hexaethylene glycol molecular weight $(\mathrm{MW})=526.7,5 \mathrm{mg} / \mathrm{mL}, 200 \mu \mathrm{L}$ as previously described by Kanaras et al. ${ }^{15} \mathrm{~A}$ freshly prepared aqueous solution of OEG was added to a solution of $13 \mathrm{~nm}$ AuNPs $(10 \mathrm{~mL}, 5 \mathrm{nM})$ while stirring. The mixture was incubated for 2 hours at room temperature, then overnight at $4^{\circ} \mathrm{C}$. OEG AuNPs were then purified by centrifugation (16,400 rpm, 15 minutes, three times), redispersed in borate buffer $(10 \mathrm{~mL}$, $0.01 \mathrm{M}, \mathrm{pH} 9$ ), and subsequently stored at $4^{\circ} \mathrm{C}$.

\section{Conjugation of peptides to OEG AuNPs}

Three different peptides (P1, P2, and P3) were conjugated to OEG AuNPs following our previously reported method. ${ }^{16}$ A peptide solution $(100 \mu \mathrm{L}, 1 \mathrm{mg} / \mathrm{mL}$, MW P1/P2=922.1, MW P3=968.2, in 0.01 M sodium borate buffer, $\mathrm{pH} 9$ ) was added to OEG AuNPs $(5 \mathrm{~mL}, 1.5 \mathrm{nM}$ in $0.01 \mathrm{M}$ sodium borate buffer, $\mathrm{pH}$ 9) followed by 1-(3-(dimethylamino) propyl)-3-ethyl-carbodiimide methiodide, $50 \mu \mathrm{L}, 0.2 \mathrm{M}$ in water and sulfo- $N$-hydroxysulfosuccinimide $(100 \mu \mathrm{L}, 0.2 \mathrm{M}$ in water). The reaction mixture was shaken for 24 hours at room temperature, subsequently purified by centrifugation (16,400 rpm, 15 minutes, three times) and redispersed in water, followed by lyophilization. The full characterization of the peptide-coated NPs has been described elsewhere. ${ }^{9,10,15,17,18}$

\section{Ex ovo CAM assay}

Fertilized eggs (total 46) were incubated at $37^{\circ} \mathrm{C}, 90 \%(\mathrm{v} / \mathrm{v})$ relative humidity, until 72 hours and gently opened into a Petri dish, allowing the yolk-sac blood vessels facing upwards. After 6 hours of incubation, which is required for the embryo stabilization, four silicone O-rings ( $8 \mathrm{~mm}$ inside and $10 \mathrm{~mm}$ outside diameter) were placed on the yolk sac (Figure 2). Inside each O-ring, $40 \mu \mathrm{L}$ of free peptides or peptideconjugated NPs were dispensed $(0.01 \mathrm{pmol} / \mu \mathrm{L}$ for peptide concentration): 1) AuNPs conjugated to P1 peptide or free $\mathrm{P} 1$ peptide, 2) AuNPs conjugated to a scrambled peptide, and 3) AuNPs conjugated to $\mathrm{P} 3$ peptide or free $\mathrm{P} 3$ peptide. The same volume of phosphate-buffered saline (PBS) was added to the remaining O-ring as control. The eggs were then incubated at $37^{\circ} \mathrm{C}, 90 \%(\mathrm{v} / \mathrm{v})$ relative humidity for 24 hours.

All the in vivo CAM experiments were performed according to the Directive 2010/63/EU of the European Parliament and of the council of 22 September 2010 on the protection of animals used for scientific purposes, which does not contain any kind of restriction to the use of non-mammal embryos. The use of this alternative animal model fits in the "3Rs policy" that was strictly followed during experiments.

Images were acquired at 0 and 24 hours of incubation using a dissecting microscope with an amplification of 19.2 times. After image acquisition, the internal area of the O-ring was analyzed using Adobe PhotoShop CS3 (ADOBE Systems Inc., San Jose, California, USA); the red, green, and blue images were then assessed in ImageJ $1.49 \mathrm{k}$ (Wayne Rasband, National Institutes of Health, USA) (Figure 1). Evaluation of images was performed as follows: first, image channels were split and the green channel was selected for further analysis. Then, noise was removed by smoothing the image (despeckling, and dark outliers with 5.0 pix with a threshold 50 were removed). For image segmentation, a noise tolerance of 8 was considered, excluding the edge maxima and binary performed (Figure 2). The skeleton plugin of ImageJ was then used for counting the number of branches within the region treated with the sample substances
A

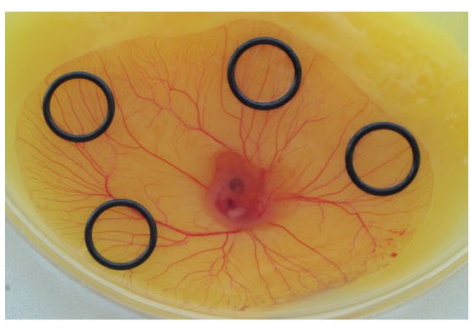

B

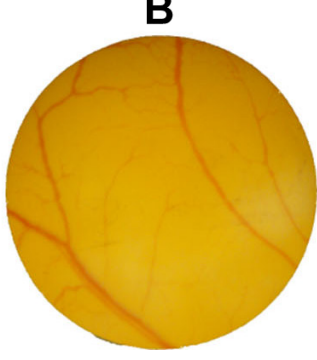

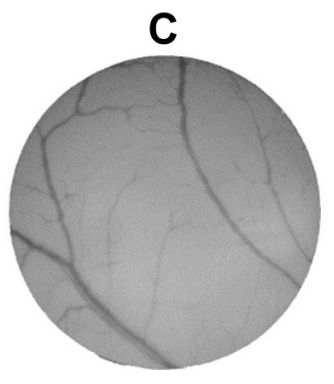

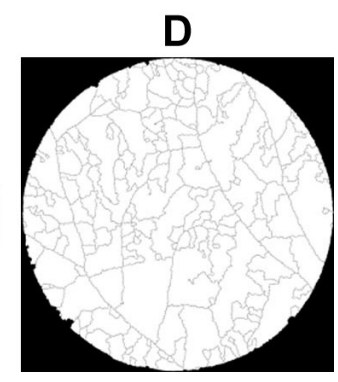

Figure 2 CAM assay

Notes: (A) Position of the silicon O-rings in the developing blood vessels plexus of the yolk sac membrane. (B) RGB image of the O-ring interior used for counting the number of veins. (C) Green channel of the same image. (D) Binary of the segmented image used to calculate the number of branches. Abbreviations: CAM, chorioallantoic membrane; RGB, red, green, and blue. 
(ie, AuNPs or free peptide). Tertiary arterioles (lowest caliber) in the image obtained after channel split were also counted manually for comparison. The percentage of tertiary arterioles (number of tertiary vessels divided by the number of branches) was calculated for both time points ( 0 and 24 hours). After normalization of the percentage of tertiary arterioles at 24 hours, compared to the initial number of tertiary arterioles ( 0 hour), the percentage of newly formed arterioles was calculated relative to the number of newly formed arterioles in the control (PBS).

\section{Statistical analysis}

Results are presented as average \pm standard deviation of triplicate experiments, unless otherwise mentioned. Differences between groups were considered significant at $P<0.05$ using multiple comparisons of two-way analysis of variance, based on Tukey's multiple comparisons test.

\section{Results and discussion Preparation of peptide OEG-coated} AuNPs

AuNPs were stabilized by OEG and subsequently conjugated to the three peptides (P1, P2, and P3) to infer their functionality as previously described (Figure 3). 9,17,18 The "activator" (P1-AuNPs), "scramble" (P2-AuNPs), and "inhibitor" (P3-AuNPs) were then incubated with chick embryos to validate the efficiency of modulating in vivo angiogenesis.

\section{Ex ovo CAM assay}

The CAM assay is generally considered a reliable in vivo model to study angiogenesis..$^{13,19}$ The percentage of the newly formed arterioles for the control group was compared to each test group, treated with either the free peptides or the AuNPs-conjugated peptides at a concentration of $0.01 \mathrm{pmol} / \mu \mathrm{L}$ (Figure 4).

The percentage of newly formed arterioles correlates well with the effect of the peptide and is a measure of the efficacy of the nanoconjugate. P3 induced a clear reduction in the formation of new arterioles when compared to the scrambled peptide (P2) $(P<0.005)$. This reduction was much more pronounced in the samples treated with P3-AuNPs $(P<0.0001)$ (Figure 5 and Table 1). On the other hand, samples treated with proangiogenic peptide (P1) clearly showed induction of new arterioles formation in both situations. Again, this effect is more evident when the CAMs are exposed to P1-AuNPs (Figure 5 and Table 1).

The in vivo results herein presented on activation and inhibition of angiogenesis corroborate our previous in vitro data using cell lines. ${ }^{9}$ The activator peptide (P1) enhanced neovascularization, and the treatment with the inhibitor peptide (P3) was able to selectively inhibit angiogenesis as observed previously in vitro. ${ }^{9}$ It is important to note here that grafting the peptides on to the surface of AuNPs provides for an enhancement of the effect of the peptide without apparent toxicity. ${ }^{9}$ This may be attributed to a focalization of the peptide within a circumscriptive region, thus allowing for increased effect. Another reason for the observed increase might be due to an NP-triggered internalization effect. As previously reported, this angiogenesis modulation occurs via a receptor-mediated process by binding to angiogenic receptors on the endothelia of forming blood vessels and/or by changing cell secretion of pro- and antiangiogenic factors. ${ }^{9}$ The enhancement of the
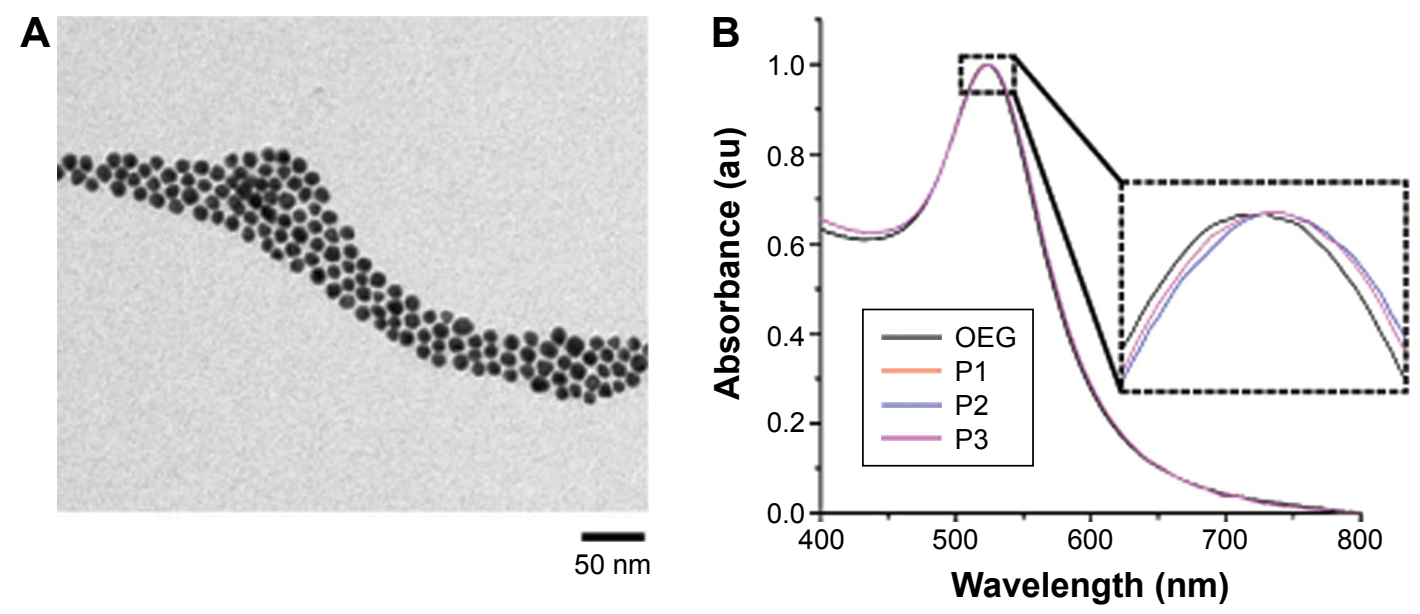

Figure 3 Characterization of peptide-coated AuNPs.

Notes: (A) Transmission electron micrograph of OEG AuNPs. (B) Normalized UV-vis spectra of OEG AuNPs and peptide-OEG AuNPs showing a slight red shift in the plasmon peak arising from a small change in the refractive index around the AuNP due to the peptide coating. Inset is magnifying the SPR peak.

Abbreviations: AuNPs, gold nanoparticles; OEG, oligo-ethylene glycol; SPR, surface plasmon resonance. 

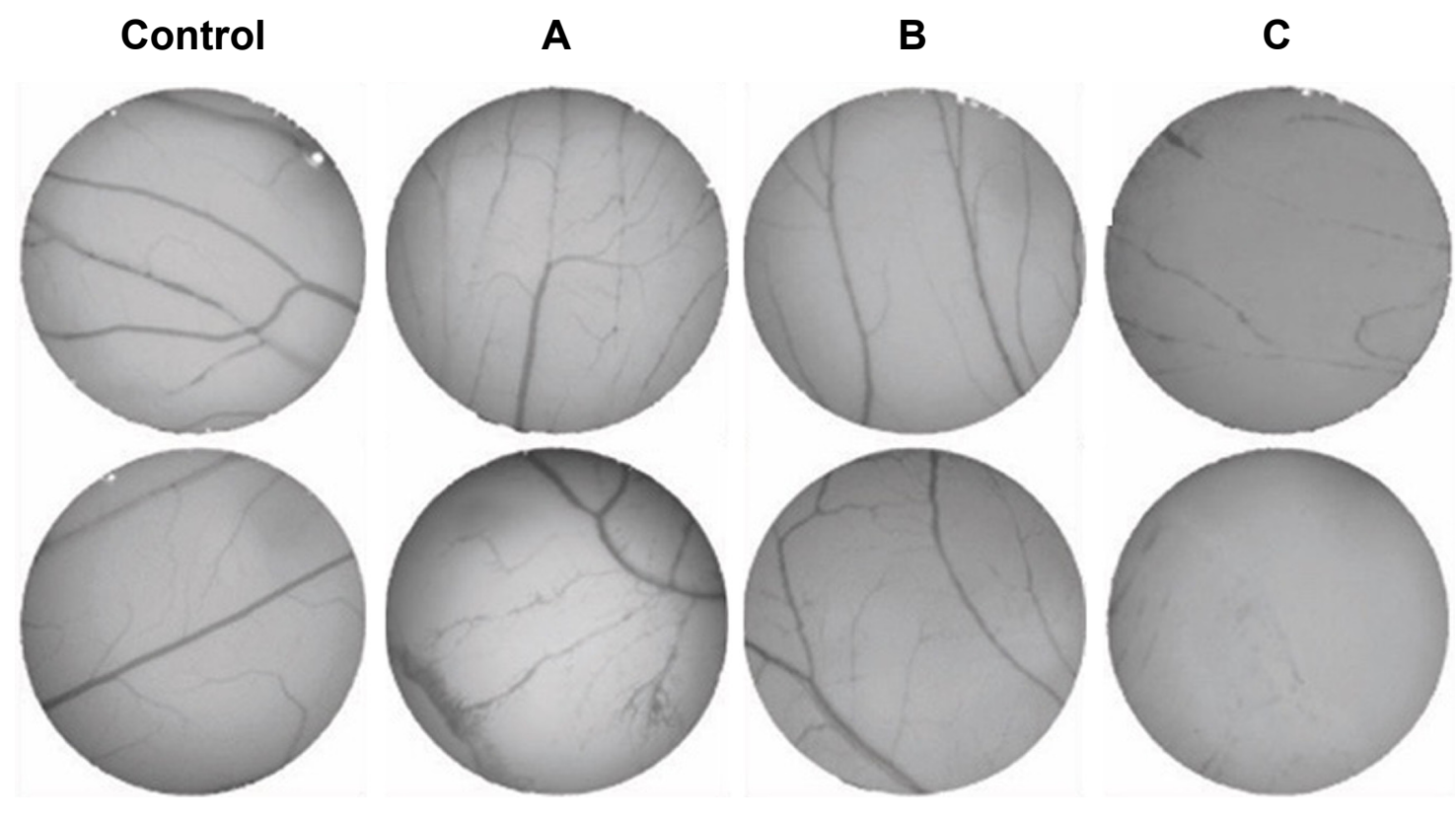

Figure 4 Angiogenesis on two independent CAMs after 24-hour exposure to: control. Notes: (A) PI-AuNPs; (B) P2-AuNPs; (C) P3-AuNPs.

Abbreviations: AuNPs, gold nanoparticles; CAM, chorioallantoic membrane.

effect of peptides conjugated to AuNPs was shown to be ruled by receptor-mediated interactions with specific receptors allocated on the target cell membrane. ${ }^{17}$ In terms of toxicity, these nanoconjugates have been previously shown not to induce acute toxicity in cell culture, ${ }^{9,17}$ which is corroborated by the lack of effect from the scramble peptide nanoconjugate when compared to PBS (P2-AuNP).

Since tumor growth and progression require vascular supply, antiangiogenic therapy is a highly effective strategy for treating cancer. ${ }^{20} \mathrm{On}$ the other end, therapies that promote

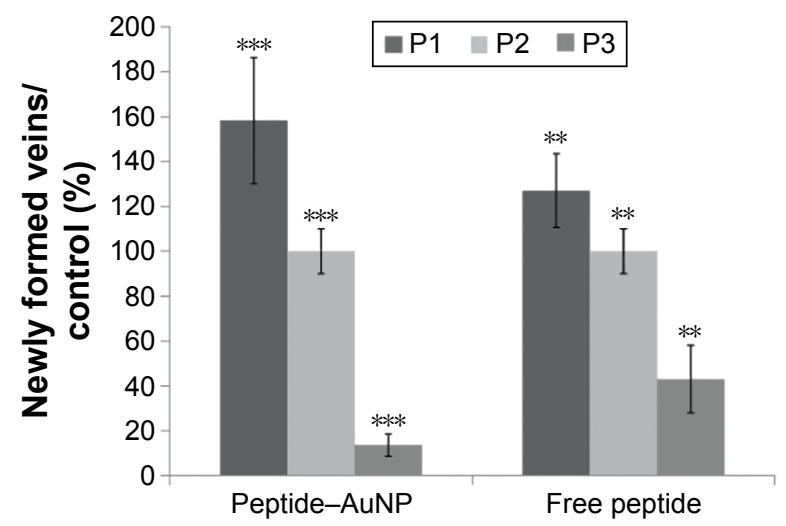

Figure 5 Percentage of newly formed arterioles.

Notes: As a result of exposure to free peptide or AuNPs (peptide-AuNPs) relative to control (phosphate-buffered saline): PI, proangiogenic peptide; P2, scrambled peptide; $\mathrm{P} 3$, antiangiogenic peptide. The concentration of peptide was kept constant $(0.01 \mathrm{pmol} / \mu \mathrm{L})$. Error bars represent the standard deviation from the mean. A total of 46 eggs were analyzed. $* * p<0.005 ; * * * p<0.0001$.

Abbreviation: AuNPs, gold nanoparticles. angiogenesis may contribute to cardiovascular regeneration, which is crucial to other disease such as diabetic cardiomyopathy and ischemic heart disease. ${ }^{21,22}$ The in vivo enhancement of angiogenesis was also observed by others using biosynthesized gold nanoconjugates from plant extracts. ${ }^{23}$ Thus, angiogenesis results from an intricate network of interactions between pro- and antiangiogenic molecules, endothelial cell receptors, and various modulators. ${ }^{24,25}$ Modulation of angiogenesis in vivo using nanovectorization systems, such as AuNPs, represents a powerful strategy not only for targeting cancer progression but also for other diseases.

\section{Conclusion}

We demonstrated for the first time the effective modulation of angiogenesis in vivo using peptide-coated AuNPs. Exposure of CAMs to peptide activators of angiogenesis accelerated the formation of new arterioles by a factor of 1.27 when compared to the scrambled peptide. Moreover, the

Table I Percentage of newly formed arterioles in CAMs exposed to free peptides (PI, P2, or P3) or the respective peptide-AuNP relative to control

\begin{tabular}{lll}
\hline Peptide & Peptide-AuNP & Free peptide \\
\hline PI & $158 \pm 28$ & $127 \pm 10$ \\
P2 & $100 \pm 16$ & $100 \pm 10$ \\
P3 & $14 \pm 4$ & $43 \pm 3$ \\
\hline
\end{tabular}

Note: Data presented as mean \pm standard deviation.

Abbreviations: AuNP, gold nanoparticle; CAM, chorioallantoic membranes. 
nanovectorization of this peptide was able to enhance neovascularization 1.58-fold. Conversely, using the same approach, inhibitors of angiogenesis were able to selectively inhibit angiogenesis in vivo. These nanovectorization systems may provide new routes of targeted delivery using multifunctional coatings and may allow additional benefits for nanotheranostics (combining imaging and treatment) applications. ${ }^{26} \mathrm{NPs}$ functionalized with KPQPRPLS or KATWLPPR peptides bind to the vascular endothelial growth factor receptor or neuropilin-1 receptor on endothelial cells for endocytosismediated internalization, promoting signal cascade and activating or inhibiting angiogenesis, respectively. ${ }^{9,26}$

We believe that these results provide a striking argument for the development of novel NP delivery systems targeting angiogenesis. Selective modulation of angiogenesis is of great interest for biomedical applications where promotion of vascular growth is desirable (eg, wound healing) or ought to be contravened, as in cancer development. It may be envisaged that AuNPs functionalized with angiogenic peptides may be used for revascularization of stroke-affected areas; conversely, antiangiogenic AuNPs may be targeted to the tumor site via the enhanced permeability and retention effect and further enhanced via active targeting moieties to tackle tumor microenvironment and prevent tumor growth by avoiding the formation of new vasculature. Simultaneously, such AuNP-based formulations will allow computed tomography imaging to evaluate tumor development in nanotheranostic strategies.

\section{Acknowledgments}

The authors acknowledge Fundação para a Ciência e a Tecnologia (FCT/MEC) for financial support (Project UID/ Multi/04378/2013). They also thank L Raposo for preliminary CAM assays.

\section{Disclosure}

The authors report no conflicts of interest in this work.

\section{References}

1. Zhao Y, Adjei AA. Targeting angiogenesis in cancer therapy: moving beyond vascular endothelial growth factor. Oncologist. 2015;20: 660-673.

2. Cabral RM, Baptista PV. Anti-cancer precision theranostics: a focus on multifunctional gold nanoparticles. Expert Rev Mol Diagn. 2014;14: 1041-1052.

3. Baptista PV. Gold nanobeacons: a potential nanotheranostics platform. Nanomedicine (London). 2014;9:2247-2250.

4. Ajith TA. Strategies used in clinical trials of gene therapy for cancer. J Exp Ther Oncol. 2015;11:33-39.

5. Sarsons CD, Tekrony A, Yaehne K, Childs S, Rinker KD, Cramb D. Testing nanoparticles for angiogenesis-related disease: charting the fastest route to the clinic. J Biomed Nanotechnol. 2014;10:1641-1676.
6. Skotland T, Iversen TG, Torgersen ML, Sandvig K. Cell-penetrating peptides: possibilities and challenges for drug delivery in vitro and in vivo. Molecules. 2015;20:13313-13323.

7. Li H, Tsui TY, Ma W. Intracellular delivery of molecular cargo using cell-penetrating peptides and the combination strategies. Int J Mol Sci. 2015;16:19518-19536.

8. Pang HB, Braun GB, Ruoslahti E. Neuropilin-1 and heparan sulfate proteoglycans cooperate in cellular uptake of nanoparticles functionalized by cationic cell-penetrating peptides. Sci Adv. 2015;1: e1500821.

9. Bartczak D, Muskens OL, Sanchez-Elsner T, Kanaras AG, Millar TM. Manipulation of in vitro angiogenesis using peptide-coated gold nanoparticles. ACS Nano. 2013;7:5628-5636.

10. Bartczak D, Muskens OL, Nitti S, Millar TM, Kanaras AG. Nanoparticles for inhibition of in vitro tumor angiogenesis: synergistic actions of ligand function and laser irradiation. Biomater Sci. 2015;3: 733-741.

11. Lokman NA, Elder AS, Ricciardelli C, Oehler MK. Chick chorioallantoic membrane (CAM) assay as an in vivo model to study the effect of newly identified molecules on ovarian cancer invasion and metastasis. Int J Mol Sci. 2012;13:9959-9970.

12. Nambiar DK, Kujur PK, Singh RP. Angiogenesis assays. Methods Mol Biol. 2016;1379:107-115.

13. Dohle DS, Pasa SD, Gustmann S, et al. Chick ex ovo culture and ex ovo CAM assay: how it really works. J Vis Exp. 2009;33:1620-1626.

14. Turkevich J, Stevenson PC, Hillier J. A study of the nucleation and growth processes in the synthesis of colloidal gold. Discuss Faraday Soc. 1951;11:55-75.

15. Kanaras AG, Kamounah FS, Schaumburg K, Kiely C, Brust M. Thioalkylated tetraethylene glycol: a new ligand for water soluble monolayer protected gold clusters. Chem Commun. 2002;20:2294-2295.

16. Bartczak D, Kanaras AG. Preparation of peptide-functionalized gold nanoparticles using one pot EDC/sulfo-NHS coupling. Langmuir. 2011;27:10119-10123.

17. Bartczak D, Sanchez-Elsner T, Louafi F, Millar TM, Kanaras AG. Receptor-mediated interactions between colloidal gold nanoparticles and human umbilical vein endothelial cells. Small. 2011;7: 388-394.

18. Bartczak D, Nitti S, Millar TM, Kanaras AG. Exocytosis of peptide functionalized gold nanoparticles in endothelial cells. Nanoscale. 2012;4: 4470-4472.

19. Ribatti D. The chick embryo chorioallantoic membrane as an in vivo assay to study antiangiogenesis. Pharmaceuticals. 2010;3:482-513.

20. Yadav L, Puri N, Rastogi V, Satpute P, Sharma V. Tumor angiogenesis and angiogenic inhibitors: a review. J Clin Diagn Res. 2015;9: XE01-XE05.

21. Saraf R, Mahmood F, Amir R, Matyal R. Neuropeptide Y is an angiogenic factor in cardiovascular regeneration. Eur J Pharmacol. 2016;776: 64-70.

22. Katoh M. Therapeutics targeting angiogenesis: genetics and epigenetics, extracellular miRNAs and signaling networks (review). Int J Mol Med. 2013;32:763-767.

23. Nethi SK, Mukherjee S, Veeriah V, Barui AK, Chatterjeeb S, Patra CR. Bioconjugated gold nanoparticles accelerate the growth of new blood vessels through redox signalling. Chem Commun. 2014;50: 14367-14370.

24. Rusnati M, Presta M. Angiogenic growth factors interactome and drug discovery: the contribution of surface plasmon resonance. Cytokine Growth Factor Rev. 2015;26:293-310.

25. Vandekeere S, Dewerchin M, Carmeliet P. Angiogenesis revisited: an overlooked role of endothelial cell metabolism in vessel sprouting. Microcirculation. 2015;22:509-517.

26. Oh N, Park J-H. Endocytosis and exocytosis of nanoparticles in mammalian cells. Int J Nanomed. 2014;9(Suppl 1):51-63. 
International Journal of Nanomedicine

Dovepress

\section{Publish your work in this journal}

The International Journal of Nanomedicine is an international, peerreviewed journal focusing on the application of nanotechnology in diagnostics, therapeutics, and drug delivery systems throughout the biomedical field. This journal is indexed on PubMed Central, MedLine, CAS, SciSearch ${ }^{\circledR}$, Current Contents ${ }^{\circledR} /$ Clinical Medicine,
Journal Citation Reports/Science Edition, EMBase, Scopus and the Elsevier Bibliographic databases. The manuscript management system is completely online and includes a very quick and fair peer-review system, which is all easy to use. Visit http://www.dovepress.com/ testimonials.php to read real quotes from published authors.

Submit your manuscript here: http://www.dovepress.com/international-journal-of-nanomedicine-journal 